\title{
MODE LOCALIZATION AND EIGENVALUE LOCI VEERING PHENOMENA IN DISORDERED STRUCTURES
}

\author{
C. Pierre \\ Department of Mechanical Engineering and Applied Mechanics, The University of Michigan, \\ Ann Arbor, Michigan, 48109-2125, U.S.A.
}

(Received 26 June 1987, and in revised form 13 May 1988)

\begin{abstract}
An investigation of the effects of disorder on the modes of vibration of nearly periodic structures is presented. It is shown that, in structures with close eigenvalues, small structural irregularities result in both strong localization of the mode shapes and abrupt veering away, or mutual repulsion, of the loci of the eigenvalues when these are plotted against a parameter representing the disorder in the system. Perturbation methods for the eigenvalue problem are applied to predict the occurrence of strong localization and eigenvalue loci veering, which are shown to be two manifestations of the same phenomenon. Also, perturbation methods that handle the dramatic effects of small disorder are developed to analyze eigenvalue loci veering and strong localization. Two representative disordered nearly periodic structures are studied: a mistuned assembly of coupled oscillators and a multi-span beam with irregular spacing of the supports.
\end{abstract}

\section{INTRODUCTION}

It is well known that the presence of small irregularities in nearly periodic structures may inhibit the propagation of vibration and localize the vibration modes. Under conditions of weak internal coupling, the mode shapes undergo dramatic changes to become strongly localized when small disorder is introduced, thereby confining the energy associated with a given mode to a small geometric region. This phenomenon, referred to as normal mode localization, has excited considerable interest in solid state physics over the years [1-5] and more recently was rediscovered in the field of structural dynamics [6-15].

To date, however, little attention has been paid to the behavior of the eigenvalues of the system when strong mode localization occurs. Interesting questions arise: Is the effect of small disorder on the eigenvalues as drastic as the one on the mode shapes? Is there a typical phenomenon exhibited by the eigenvalues corresponding to mode localization? The present study is an attempt to provide answers to these questions.

Generally speaking, the dependence of the eigenvalues upon a system parameter is often of interest in structural dynamics. This leads to a group of loci when the eigenvalues are plotted versus the system parameter. Such eigenvalue loci are known to have fascinating characteristics, which were first discovered by Leissa in a pioneer paper [16]. When two eigenvalue loci approach each other, they either cross or do not cross. Often in the latter case, even though the loci nearly intersect, in fact they do not but rather veer away from each other with high local curvature. This phenomenon, referred to as eigenvalue loci veering, or curve veering, has been thoroughly studied in both dynamics and physics [16-19]. Curve veering was first thought by Leissa to be a phenomenon created by the discretization of continuous systems. However, in a recent paper, Perkins and Mote [19] showed by a perturbation approach that curve veering indeed occurs in continuous as well as in discrete systems. 
It has also been observed by Leissa and others [16-18] that when eigenvalue loci veering occurs, the eigenfunctions undergo dramatic, albeit continuous, changes in the veering region. Indeed, the eigenfunctions corresponding to each eigenvalue locus are interchanged during veering. However, to date, the occurrence of eigenvalue loci veering has never been associated with the occurrence of strong mode localization.

Both eigenvalue loci veering and mode localization are catastrophic type phenomena, because small changes in the system parameters result in large variations in the eigenvalues and the mode shapes, respectively. Since both phenomena occur when a particular system parameter is varied (for mode localization, this parameter is a measure of disorder), one wonders whether the occurrence of these two phenomena might be related in any way.

This paper describes an investigation of the effects of small disorder on the modes of free vibration of nearly periodic structures. Special attention is paid to the loci of the eigenvalues versus a parameter representing the amount of disorder in the system. It is shown that when small disorder is introduced in conservative nearly periodic structures with weak internal coupling, both strong mode localization and veering of the eigenvalue loci occur, indicating that these are two manifestations of the same phenomenon. This simultaneous occurrence is illustrated in section 2 with a simple two-degree-of-freedom (DOF) system. Section 3 presents perturbative approaches for both the prediction and analysis of mode localization and curve veering in self-adjoint disordered systems. In sections 4 and 5 the general theory is applied to two simple disordered systems, namely an assembly of coupled oscillators and a two-span beam whose localization of the modes has been previously studied by the author $[12,13]$. The (modified) perturbation method developed in references $[12,13]$ for the analysis of strong mode localization is shown to apply to the analysis of curve veering as well.

\section{MODE LOCALIZATION AND CURVE VEERING-A SIMPLE EXAMPLE}

The system of two coupled oscillators shown in Figure 1 is considered. The free vibration eigenvalue problem is given in Appendix $\mathrm{A}$ for $N=2, \Delta l_{1}=0$, and $\Delta l_{2}=\Delta l$. The two important parameters are the dimensionless coupling between pendulums, $R^{2}=$ $(k / m) /(g / l)$, and the dimensionless length deviation, $\Delta l$. For $\Delta l=0$, the system is tuned, or ordered; otherwise, it is mistuned, or disordered. The modes of vibration of the tuned system are given in Appendix A.

Figure 2 represents the loci of the two dimensionless eigenvalues versus disorder, $\Delta l$. Mode shapes of angular amplitudes are also displayed for both tuned and mistuned systems. Figure 2(a) is for a strongly coupled system such that $R=0 \cdot 5$. Observe that small disorder does not have much effect on both the eigenvalues and the mode shapes, as the modes of the mistuned system are merely perturbations of those of the tuned system. Also note that the two eigenvalues are far apart. Now consider Figure 2(b), which is for weak coupling, $R=0.025$. While the mode shapes of the ordered system are still extended (in fact, this is the case for arbitrarily small coupling), the modes of the

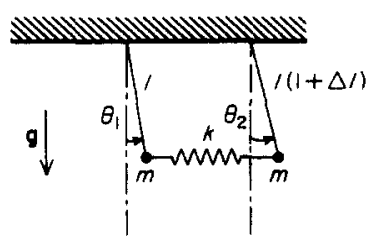

Figure 1. Two coupled oscillators. 


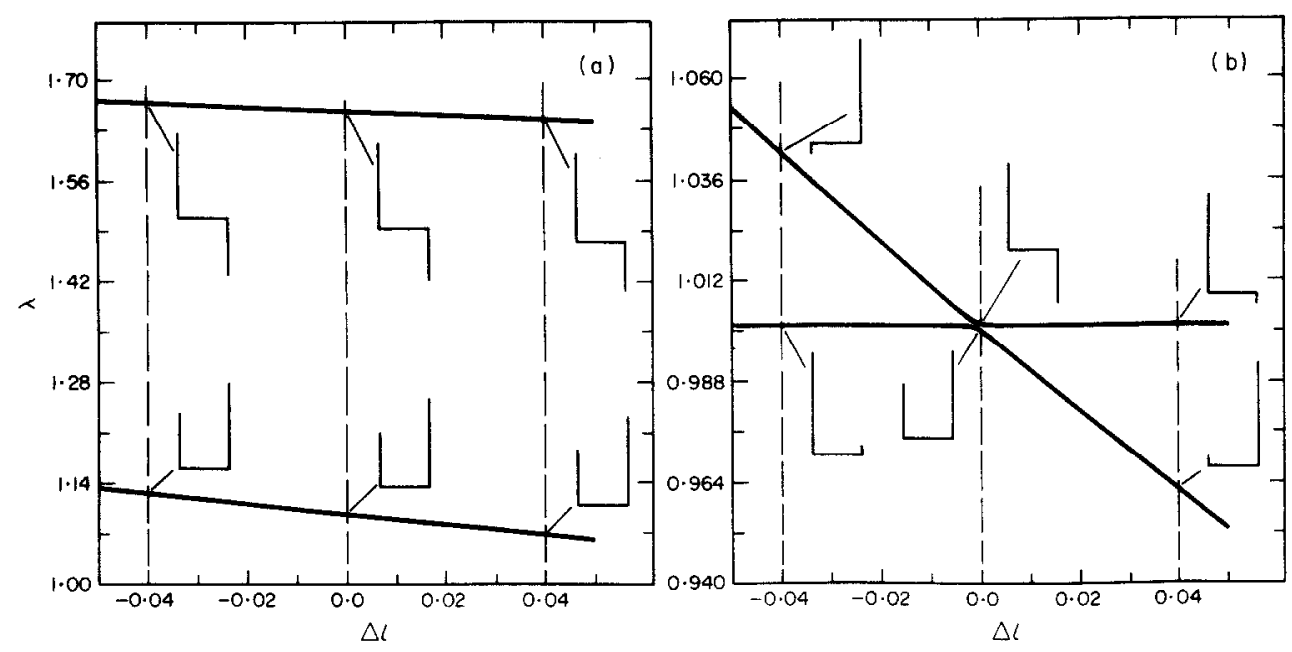

Figure 2. Loci of the dimensionless eigenvalues of the two-pendulum system in terms of the disorder, $\Delta l$; representative mode shapes are shown. (a) The strong interpendulum coupling case, $R=0.5$; neither eigenvalue loci veering nor mode localization occur. (b) The weak interpendulum coupling case, $R=0.025$; both curve veering and strong localization occur.

mistuned system become strongly localized about one pendulum when small disorder is introduced, a well-known phenomenon [6]. Indeed, these localized modes are perturbations of those of the decoupled mistuned system, not of those of the tuned system. Perhaps less expected, the hehavior of the eigenvalues in Figure $2(b)$ is also quite different from that in Figure 2(a). One observes that for small coupling the loci seem to cross at the ordered state, although in fact they do not cross but veer abruptly from each other with high local curvatures. The loci cannot cross because there is no multiple eigenvalue for the system, which results in a mutual repulsion of both loci, or curve veering. For strong interpendulum coupling no such drastic phenomenon is observed. One concludes that both strong mode localization and eigenvalue loci veering occur for weak coupling between oscillators, that is, when the eigenvalues of the ordered system are close. This simultaneous occurrence is generalized in the next section.

\section{PERTURBATIVE ANALYSIS OF DISORDERED STRUCTURES}

\subsection{PREDICTION OF STRONG MODE LOCALIZATION}

Even though classical perturbation methods fail to describe localization quantitatively because the localized modes are dramatically different from the unperturbed (extended) ones, they provide useful insight into the onset of localization. (The reader not familiar with perturbation theory for the eigenvalue problem is referred to references [20] and [21].) Consider an unperturbed (i.e., ordered) system represented by an operator $L_{0}$ (a homogeneous linear differential operator for a continuous system or an $n \times n$ matrix for a discrete system). The free vibration eigenvalue problem is

$$
L_{0} w_{0 i}=\lambda_{0 i} w_{0 i}, \quad i=1, \ldots,
$$

where $\left(\lambda_{0 i}, w_{0 i}\right)$ is an unperturbed eigensolution. For a discrete system, there are $n$ $n$-dimensional eigenvectors, while for a continuous system, $\dagger$ there is a countable infinity

† The function space considered is the space of comparison functions [20]; thus all boundary conditions are satisfied. 
of eigenfunctions of the space variables. For simplicity of presentation, a standard eigenvalue problem is considered, the eigenvalues are assumed to be simple, and $L_{0}$ is taken to be self-adjoint, although this can be generalized.

Denote by $p$ the parameter(s) through which disorder is introduced into the structure. $p$ can be a vector for a discrete system (e.g., the vector of pendulum lengths for a chain of pendulums) or a function of the space variable(s) for a continuous system. Let $p_{0}$ be the unperturbed value to which corresponds $L_{0} \equiv L\left(p_{0}\right)$. The introduction of disorder, or mistuning, perturbs $p_{0}$ as

$$
p=p_{0}+d p=p_{0}+\bar{p} \varepsilon, \quad\|\bar{p}\|=1,
$$

where $d p$ accounts for the structural parameter perturbations. In absolute value, the perturbation parameter $\varepsilon$ equals the Euclidean norm of $d p$. Note that $\varepsilon$ could also have been defined as an estimate of the standard deviation of the perturbations from the mean (as is chosen in section 4). The operator $L_{0}$ is accordingly perturbed as

$$
L(p) \equiv L=L_{0}+d L=L_{0}+l \varepsilon+m \varepsilon^{2}+\cdots,
$$

where $d L$ is a perturbation operator that includes terms of the first and higher order in the parameter perturbations, $d p$. The (operator) norms of $l$ and $m$ are finite, on the order of one, and the norm of $d L$ is first order, of order $\varepsilon$.

The eigensolution is accordingly perturbed to the second order as

$$
\lambda_{i}=\lambda_{0 i}+\delta \lambda_{i}+\delta^{2} \lambda_{i}, \quad w_{i}=w_{0 i}+\delta w_{i}+\delta^{2} w_{i},
$$

where $\delta \lambda_{i}$ and $\delta w_{i}$ (resp. $\delta^{2} \lambda_{i}$ and $\delta^{2} w_{i}$ ) are first- (resp. second-) order terms in $\varepsilon$. One can show $[12,20,21]$ that the first-order perturbations are given by

$$
\delta \lambda_{i}=\left\langle w_{0 i}, l w_{0 i}\right\rangle \varepsilon, \quad \delta w_{i}=\left\{\sum_{j \neq i} \frac{\left\langle w_{0 j}, l w_{0 i}\right\rangle}{\lambda_{0 i}-\lambda_{0 j}} w_{0 j}\right\} \varepsilon
$$

where $\langle\cdot, \cdot\rangle$ denotes an inner product defined by $\langle u, v\rangle=\int_{\Omega} u v \mathrm{~d} \Omega$ for a continuous system, where $\Omega$ is the domain of the system. For a discrete system, $\langle u, v\rangle=u^{\mathrm{T}} v$ is a vector scalar product, where $\mathrm{T}$ denotes a transpose. The unperturbed eigenfunctions are normalized as $\left\langle w_{0 k}, w_{0 l}\right\rangle=\delta_{k}^{l}$, where $\delta_{k}^{l}$ is the Kronecker symbol.

Note that $\delta \lambda_{i}$ is always a first-order term that is a measure of the first order perturbation operator $l \varepsilon$. However, it is not a good measure of the amount of disorder, as if $l$ is proportional to $L_{0}$ for instance (i.e., no disorder), $\left\langle w_{0 i}, l w_{0 i}\right\rangle$ does not vanish. On the other hand, the magnitude of $\delta w_{i}$ is determined by the values of the ratios $\left(w_{0 j}, l w_{0 i}\right) \varepsilon /\left(\lambda_{0 i}-\lambda_{0 j}\right)$. If all the eigenvalues are well separated, then $\delta w_{i}$ is effectively first-order. However, if two eigenvalues, say $\lambda_{0 r}$ and $\lambda_{0 s}$, are close, such that $\left|\lambda_{0 r}-\lambda_{0 s}\right|$ is of the order of or smaller than $\left|\left\langle w_{0 s}, l w_{0 r}\right\rangle \varepsilon\right|$, then $\delta w_{r}$ and $\delta w_{s}$ are not first-order any longer, but of the order of one or larger. The assumptions for the use of asymptotic expansions in perturbation theory are then violated, and the perturbation analysis fails, thereby indicating that the modes undergo a dramatic change. Indeed, for disordered structures made of coupled component systems, the failure of the perturbation analysis indicates the occurrence of strong localization, as follows.

For the system of coupled oscillators shown in Figure 3 [12], the distance between the unperturbed eigenvalues is proportional to the coupling between oscillators, $R^{2}$ (see equation (A2)). Similarly, for the two-span beam shown in Figure 4, the distance between the eigenvalues of a pair of modes is also a measure of the coupling between spans, as it is proportional to $1 / c$ for large $c$, where $c$ is the stiffness constant of the torsional spring at the intermediate support [13]. More recently, it was shown by the author [14] that for nearly periodic structures made of coupled component systems, the eigenvalues are 

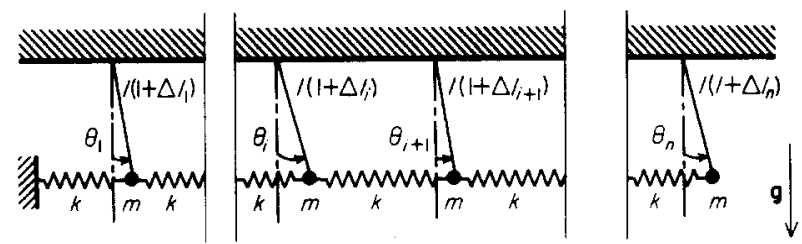

Figure 3. Disordered chain of $n$ coupled oscillators.

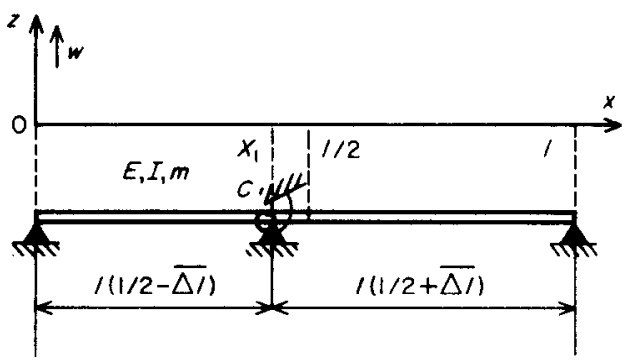

Figure 4. Geometry of disordered two-span beam.

clustered in groups whose width decreases with the coupling between the component systems. Therefore, in periodic structures made of coupled component systems, $\left|\lambda_{0 r}-\lambda_{0 s}\right|$ is a measure of the internal coupling. Moreover, $\left\langle w_{0 r}, l w_{0 s}\right\rangle \varepsilon$ is a measure of the disorder, where $\varepsilon$ indicates its magnitude and $\left\langle w_{0 r}, l w_{0 s}\right\rangle$ its spatial distribution. For example, if $l$ is proportional to $L_{0}$ (no disorder), then $\left\langle w_{0 r}, l w_{0 s}\right\rangle=0$.

Previous studies [6-14] have shown that strong localization occurs in structures made of coupled component systems when the internal coupling is of the order of or smaller than the disorder. The above discussion indicates that an equivalent criterion for localization is when the distance between two of the unperturbed eigenvalues is of the order of or smaller than the inner product of the corresponding eigenfunctions through the operator perturbation:

$$
\left|\lambda_{0 r}-\lambda_{0 s}\right| \leqslant O\left(\left|\left\langle w_{0 s}, l w_{0 r}\right\rangle \varepsilon\right|\right)
$$

The occurrence of strong localization is then indicated by the failure of the perturbation analysis. Note that if $\left\langle w_{0 s}, l w_{0 r}\right\rangle=0$ (e.g., no disorder in the system), the criterion (7) predicts that the perturbation analysis is valid no matter how close the eigenvalues are, and thus that no strong localization occurs-a consistent result.

Therefore, there are two key parameters involved in the occurrence of localized modes. The first one is the spacing between the eigenvalues of the ordered system $[6,12]$. The second parameter, rather than the perturbation $l \varepsilon$, is the coupling of the unperturbed eigenfunctions through the operator perturbation. Finally, note that, for nearly periodic structures made of coupled component systems, two "couplings" are involved in the occurrence of localization. The first is a physical coupling between component systems, which determines the distance between eigenvalues, and the second is the coupling between mode shapes through the parameter perturbations, with localization occurring if the former is of the order of or smaller than the latter.

\subsection{EIGENVALUE LOCI VEERING}

While the first-order perturbation formulae (5) and (6) provide insight into the occurrence of localization, interesting and useful behavior can also be observed by considering 
the second-order eigenvalue perturbations $[11,21]$ :

$$
\delta^{2} \lambda_{i}=\sum_{j \neq i} \frac{\left\langle w_{0 j}, l w_{0 i}\right\rangle\left\langle w_{0 j}, l^{*} w_{0 i}\right\rangle}{\lambda_{0 i}-\lambda_{0 j}} \varepsilon^{2}+\left\langle w_{0 i}, m w_{0 i}\right\rangle \varepsilon^{2}
$$

where * denotes the adjoint of an operator. If all the eigenvalues are well separated, then $\delta^{2} \lambda_{i}$ is indeed second order. However, if two eigenvalues $\lambda_{0 r}$ and $\lambda_{0 s}$ are close, such that $\left|\lambda_{0 r}-\lambda_{0 s}\right|$ is on the order of or smaller than $\left\langle w_{0 s}, l w_{0 r}\right\rangle \varepsilon$, then $\delta^{2} \lambda_{r}$ and $\delta^{2} \lambda_{s}$ are of order $\varepsilon$ or larger, which violates the assumptions of perturbation methods. Thus, for systems with close unperturbed eigenvalues, the failure of the perturbation approach is indicated by both the first-order perturbation of the eigenfunctions and the second-order perturbation of the eigenvalues. Note that since the first-order eigenvalue perturbations always remain well behaved (of order $\varepsilon$ ), a first-order sensitivity analysis may completely overlook the drastic changes due to irregularities, hence, the importance of systematically performing a second-order eigenvalue sensitivity analysis.

Now, examine the loci of the eigenvalues versus a parameter representing the disorder in the system, $\varepsilon$. A second-order expansion of the $i$ th eigenvalue locus in the neighborhood of the ordered state is

$$
\lambda_{i}=\lambda_{0 i}+\left\langle w_{0 i}, l w_{0 i}\right\rangle \varepsilon+\left\langle w_{0 i}, m w_{0 i}\right\rangle \varepsilon^{2}+\sum_{j \neq i} \frac{\left\langle w_{0 j}, l w_{0 i}\right\rangle\left\langle w_{0 j}, l^{*} w_{0 i}\right\rangle}{\lambda_{0 i}-\lambda_{0 j}} \varepsilon^{2}+O\left(\varepsilon^{3}\right)
$$

The slope of the $i$ th locus at the ordered state $(\varepsilon=0)$ is $\partial \lambda_{i} /\left.\partial \varepsilon\right|_{0}=\left\langle w_{0 i}, l w_{0 i}\right\rangle$, always a well-behaved term, while the curvature of the locus at $\varepsilon=0$ is

$$
C_{i}=\frac{\left.\left(\partial^{2} \lambda_{i} / \partial \varepsilon^{2}\right)\right|_{0}}{\left(1+\left(\partial \lambda_{i} /\left.\partial \varepsilon\right|_{0}\right)^{2}\right)^{3 / 2}}=\frac{2}{\left(1+\left\langle w_{0 i}, l w_{0 i}\right\rangle^{2}\right)^{3 / 2}}\left\{\sum_{j \neq i} \frac{\left\langle w_{0 j}, l w_{0 i}\right\rangle\left\langle w_{0 j}, l^{*} w_{0 i}\right\rangle}{\lambda_{0 i}-\lambda_{0 j}}+\left\langle w_{0 i}, m w_{0 i}\right\rangle\right\},
$$

whose magnitude is determined by the distance between the unperturbed eigenvalues. If two eigenvalues, $\lambda_{0 r}$ and $\lambda_{0 s}$, are close such that their difference is on the order of or smaller than $\left\langle w_{0 s}, l w_{0 r}\right\rangle \varepsilon$, then the curvatures $C_{r}$ and $C_{s}$ of the loci of $\lambda_{r}$ and $\lambda_{s}$ are of order $1 / \varepsilon$ or larger in the neighborhood of the ordered state of the system. Now consider $\lambda_{0 r}$ and $\lambda_{0 s}$ to be the smallest and largest eigenvalues of a group of eigenvalues. If $l$ is self-adjoint, and taking $\lambda_{0 r}<\lambda_{0 s}$, one observes from equation (8) that $\delta^{2} \lambda_{r}$ is negative, while $\delta^{2} \lambda_{s}$ is positive. Thus, from equation (10), the curvature of the locus of $\lambda_{r}$ is negative, while the one of $\lambda_{s}$ is positive, both being large. One concludes that the two loci abruptly veer away from each other, as sketched in Figure 2(b). Hence, if some unperturbed eigenvalues are close, the introduction of irregularities may, in terms of eigenfunctions, localize the vibration modes and, in terms of eigenvalues, make the corresponding loci veer away rapidly with high local curvature. Moreover, if the "coupling" $\left\langle w_{0 s}, l w_{0 r}\right\rangle \varepsilon$ is equal to zero (or one or more orders of magnitude smaller than $\left.\left|\lambda_{0 r}-\lambda_{0 s}\right|\right)$, then $\delta^{2} \lambda_{r}$ and $\delta^{2} \lambda_{s}$ are second order and the loci do not veer away. One also notes from equation (7) that in this case no strong localization occurs, a consistent result.

Since they obey the same criterion(7), eigenvalue loci veering and strong mode localization are indeed two manifestations of a single phenomenon occurring in disordered systems. Therefore, the investigation of the loci of the eigenvalues in the neighborhood of the ordered state is sufficient for determining the occurrence of strong localization. A second-order eigenvalue sensitivity analysis can be used as a simple criterion for localization.

\subsection{ANALYSIS OF CURVE VEERING AND LOCALIZATION BY MODIFIED PERTURBATION METHODS}

Once mode localization and curve veering have been predicted to occur by the perturbative approach described above, the next step is to analyze the characteristics of 
the eigenvalue loci and localized modes of the disordered system. To do so, perturbation methods have to be modified to handle the dramatic changes resulting from small irregularities. Such an approach has been developed in references $[12,13]$ to analyze localization in chains of coupled oscillators (Figure 3) and two-span beams (Figure 4). For both systems, the (small) coupling between component systems, which governs the (small) distance between eigenvalues, is treated as a perturbation while the parameter irregularities are included in the unperturbed system. This approach has been generalized to nearly periodic structures made of coupled component systems in reference [14]. The extension of the method to the analysis of eigenvalue loci veering is illustrated in the next sections and in the appendices.

Recall that the classical perturbation procedure fails when ratios such as $\left\langle w_{0 j}, l w_{0 i}\right\rangle \varepsilon /\left(\lambda_{0 i}-\lambda_{0 j}\right)$ are of order one or larger. On the other hand, the modified method is defined by considering the (small) distance between the eigenvalues of the ordered system (or the width of the eigenvalue cluster) as a perturbation, while disorder (represented by $d L$ ) is included in the unperturbed system in order to split the (modified) unperturbed eigenvalues; thus, the modified perturbation method makes quantities appear similar to the (small) inverse of these ratios in the eigensolution perturbations, and therefore is particularly well adapted to the treatment of close eigenvalues and thus of localized modes and of loci presenting veerings. However, even though it detects the change in direction of the loci, the modified approach fails for very small disorder (of order $\varepsilon^{2}$ or smaller), that is, in the veering region, in which case one must use a classical procedure. Therefore, both procedures complement each other.

\section{EXAMPLE 1-CHAIN OF COUPLED OSCILLATORS}

The equations of motion for the assembly of coupled pendulums shown in Figure 3 are given in Appendix A. Two important parameters are the dimensionless coupling between pendulums, $R^{2}$, and the dimensionless length deviation from the nominal value for the $i$ th pendulum, $\Delta l_{i}$. The vector of parameter perturbations is $\Delta \mathbf{l}=$ $\left[\Delta l_{1}, \ldots, \Delta l_{i}, \ldots, \Delta l_{n}\right]^{\mathrm{T}}$. The mean of the $\Delta l_{i}$ 's is taken to be zero; thus the absolute value of the parameter $\varepsilon$ (defined by equation (2) as the norm of $\Delta \mathbf{l}$ ) is also an estimate of the standard deviation of the irregularities multiplied by $\sqrt{n}$. Here, the eigenvalues are to be plotted versus the parameter $\varepsilon^{\prime}=\varepsilon / \sqrt{n}$, whose absolute value is the standard deviation of the irregularities, $\sigma$, and thus is a good measure of disorder. In order to study the loci of the eigenvalues, the amplitude of the mistuning distribution, $\varepsilon^{\prime}$, is varied for a given random-like pattern of mistuning. One can write

$$
\Delta \mathbf{l}=\overline{\mathbf{I}} \boldsymbol{\varepsilon}^{\prime}
$$

where $\overline{\mathbf{l}}$, listed in Table 1 , has a standard deviation of 1.0 and $\varepsilon^{\prime}$ can be negative. Even though such a deterministic calculation cannot be substituted for a probabilistic treatment of disorder, it is believed to describe the behavior of the system representatively.

The loci of the eigenvalues of a chain of six coupled pendulums versus mistuning strength are shown in Figure 5(a) for strong coupling, $R=0 \cdot 5$. No curve veering is

TABLE 1

The components of the vector $\mathbf{I}$

$$
\begin{array}{ll}
\bar{l}_{1}=1.341 & \bar{l}_{4}=-0.146 \\
\bar{l}_{2}=0.610 & \bar{l}_{5}=-1.293 \\
\bar{l}_{3}=-0.976 & \bar{l}_{6}=0.439
\end{array}
$$




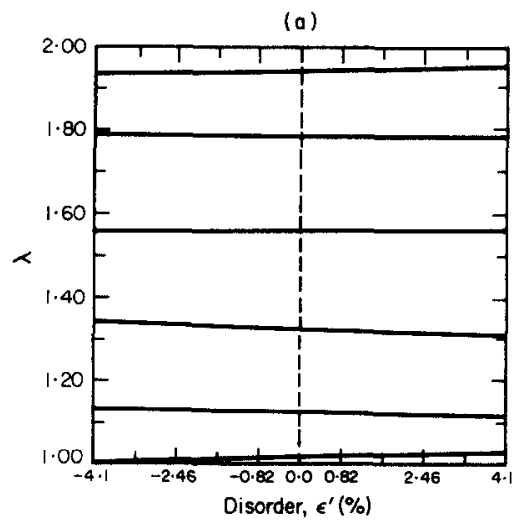

(b)
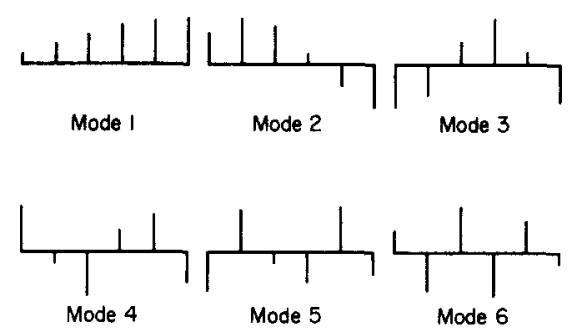

(c)
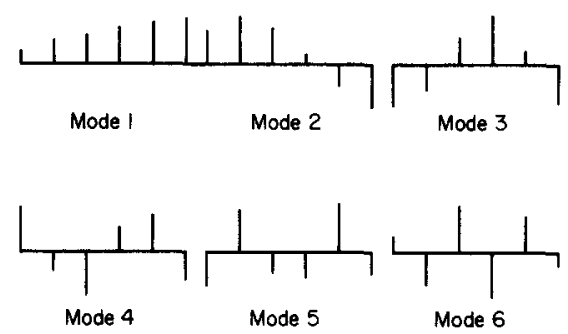

Figure 5. (a) Loci of the dimensionless eigenvalues of a chain of six strongly coupled pendulums, for $R=0 \cdot 5$, versus disorder parameter $\varepsilon^{\prime}$; no loci veering occurs; both exact method and classical perturbation method results are shown. (b) Mode shapes of an ordered assembly of six pendulums. (c) Mode shapes of a disordered chain of six strongly coupled pendulums, for $R=0.5$ and $\varepsilon^{\prime}=3 \%$.

observed. The loci on Figure 5(a) have been obtained by both the exact method and the (classical) perturbation method for strong coupling case outlined in Appendix A. The modes of the tuned and mistuned systems are displayed in Figures 5(b) and $5(\mathrm{c})$ for $\sigma=\left|\varepsilon^{\prime}\right|=3 \%$. The tuned modes are sinusoidal in space, and the mistuned modes are merely perturbations of the tuned ones. Hence, there is no significant effect of irregularities in the strong coupling case.

The eigenvalue loci versus mistuning are displayed in Figure 6(a) for weak interpendulum coupling, $R=0 \cdot 05$. One observes that the eigenvalues globally veer away from each other in the neighborhood of the tuned state, $\varepsilon^{\prime}=0$. Veering away is most pronounced for the smallest and largest eigenvalues. Note that in the small coupling case the tuned eigenvalues are very close, and thus, the abrupt veering away of the loci is expected. However, curve veering was shown to occur in section 3.2 for self-adjoint perturbations only, while for the pendulum system the (classical) perturbation matrix is not symmetric (equation (A3)). A close look at the perturbation matrix, though, reveals that for small 


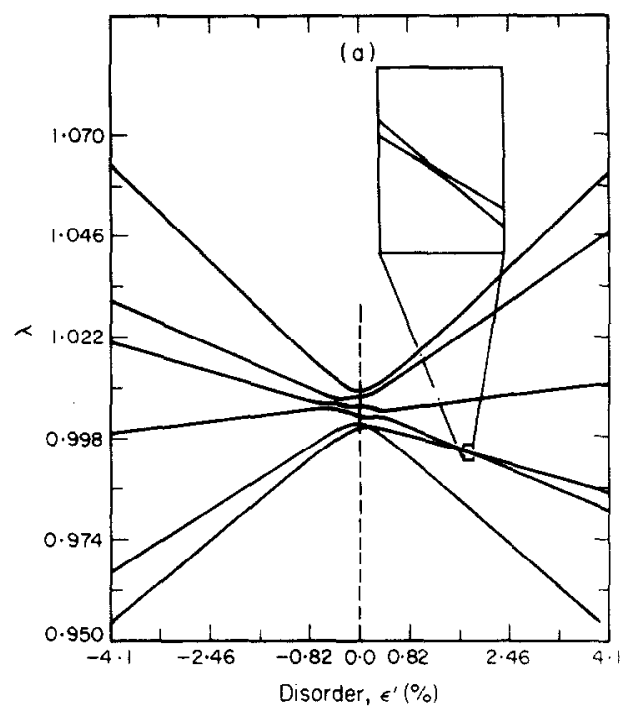

(b)

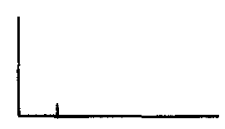

Mode 1

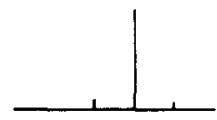

Mode 4

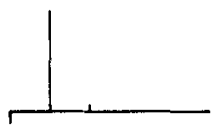

Mode 2

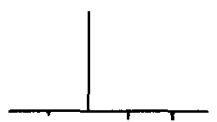

Mode 5

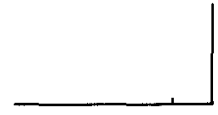

Mode 3

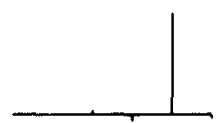

Mode 6

Figure 6. (a) Loci of the eigenvalues of a chain of six weakly coupled pendulums, for $R=0 \cdot 05$, versus disorder $\varepsilon^{\prime}$; strong curve veering occurs. (b) Mode shapes of a disordered chain of six weakly coupled pendulums, for $R=0.05$ and $\varepsilon^{\prime}=3 \%$.

(first-order) coupling the symmetric part of the matrix is first order, while the skewsymmetric part is second-order. Therefore, the perturbation matrix is essentially selfadjoint, and loci veering can be predicted by the approach of section 3.2.

The modes of the mistuned system with weak coupling are shown in Figure 6(b) for $\sigma=\varepsilon^{\prime}=3 \%$. Observe that the modes are strongly localized about one pendulum-a drastic change from the extended tuned modes. Therefore, as predicted in section 3, both mode localization and eigenvalue veering occur. Both phenomena become more pronounced as the interpendulum coupling decreases, because the distance between eigenvalues decreases with $R^{2}$ and the curvature of the loci is proportional to $1 / R^{2}$.

Note that besides the global veering of the eigenvalue loci, local veerings also occur. Since this system has only simple eigenvalues, two loci converging toward each other can only veer away and interchange their slope, instead of crossing. On Figure 6(a), one of these local veerings can be easily mistaken for a crossing, and expensive enlargements are required to exhibit the veering. Therefore, veerings and crossings can be very difficult to distinguish, as noted in reference [19].

An interesting special case is the one of decoupled pendulums, $R=0$. Since the tuned system has an $n$-fold multiple eigenvalue, the eigenvalue loci cross at $\varepsilon=0$. For arbitrarily 
small mistuning, though, the modes of the system become completely localized, as they consist of oscillations of decoupled pendulums at slightly different frequencies. Moreover, for $\boldsymbol{R}=\mathbf{0}$ and arbitrarily small (but non-zero) mistuning, the eigenvector coupling through the parameter perturbation can be readily seen in Appendix A to be equal to zero. (Here the unperturbed system must be slightly mistuned as the tuned system has a multiple eigenvalue for which the perturbation expansions (6) and (8) are not valid.) Therefore, the curvatures of the eigenvalue loci, as well as the eigenvector perturbations, are equal to zero. This enables the loci to cross locally in a linear fashion, as seen in Figure 7.

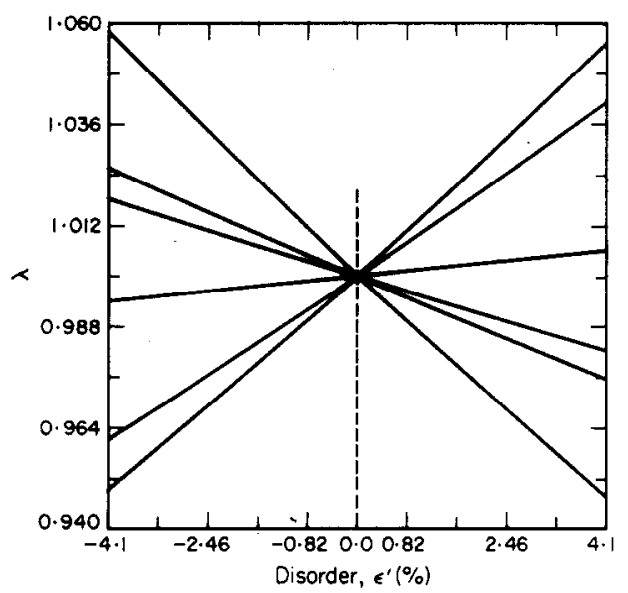

Figure 7. Loci of the dimensionless eigenvalues of a chain of six decoupled pendulums $(R=0)$ versus disorder $\varepsilon^{\prime}$. Linear crossing occurs.

Curve veering has also been analyzed by both classical and modified perturbation methods outlined in Appendix A (see reference [12] for details). Figure 8 compares the loci of the smallest and largest eigenvalues in the weak coupling case by the exact method and the classical perturbation method. As shown in section 3.2, the first-order calculation predicts a smooth linear variation of the loci that completely misses the veering and, therefore, the high sensitivity of the system. The second-order calculation, represented

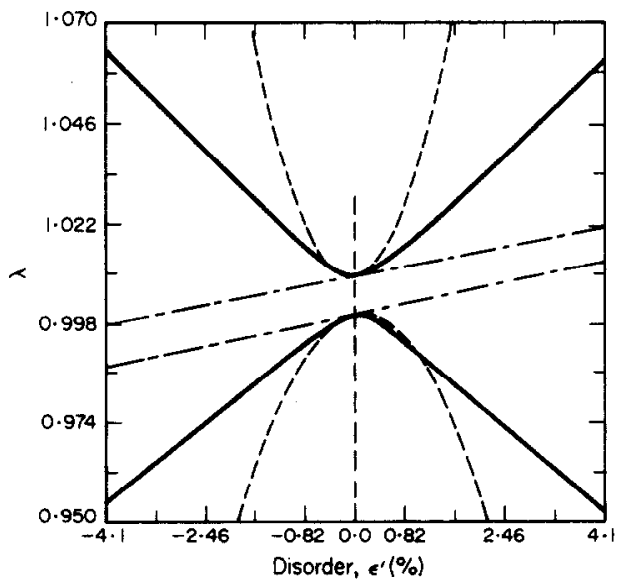

Figure 8. Loci of the smallest and largest eigenvalues of a chain of six weakly coupled pendulums $(R=0.05)$ versus disorder $\varepsilon^{\prime}$, by both the exact method (_- ) and the first- (_- -$)$ and second-order (- - $)$ classical perturbation methods. 
by a tangent parabola, detects the large curvature of the loci and therefore curve veering and localization. It is only valid in the veering region (for very small mistuning, of order $\varepsilon^{2}$ ), though, and does not describe the loci for small mistuning (of order $\varepsilon$ ). But while the first-order analysis can be dangerously misleading, the second-order analysis is useful since it indicates the high sensitivity of the system and describes the loci near the veering.

The modified perturbation approach developed in reference [12] to analyze localized modes has been extended to deal with eigenvalues. Recall that the disorder is included in the (modified) unperturbed system, while the perturbation consists of the interpendulum coupling. From Appendix A, the first- and second-order modified perturbations of the eigenvalues can be shown to be

$$
\lambda_{0 i}^{m}=1-\Delta l_{i}, \quad \delta \lambda_{i}^{m}=2 R^{2}-\delta_{i}^{n} R^{2}, \quad \delta^{2} \lambda_{i}^{m}=R^{4}\left[\frac{1-\delta_{i}^{1}}{\Delta l_{i-1}-\Delta l_{i}}+\frac{1-\delta_{i}^{n}}{\Delta l_{i+1}-\Delta l_{i}}\right]+\Delta l_{i}^{2} .
$$

These modified perturbation results are compared to the exact solution in Figure 9 in the weak coupling case. Both smallest and largest eigenvalues are plotted. For "not too small" mistuning (of order $\varepsilon$ ), i.e., when strong localization occurs, the first-order calculation is a fair approximation of the exact loci, while the second-order analysis agrees very well with the exact results. In particular, the modified procedure detects the change in slope of the loci. Note, however, that it cannot be used in the veering region where mistuning is too small (of order $\varepsilon^{2}$ or smaller): that is, too small for strong localization to occur. Indeed, for very small $\varepsilon^{\prime}(<0 \cdot 3 \%)$, the second-order modified perturbation terms become very large as $\varepsilon^{\prime}$ goes to zero, as can be seen from equation (12). In this case, however, the classical perturbation method can be used effectively-see Figure 8.

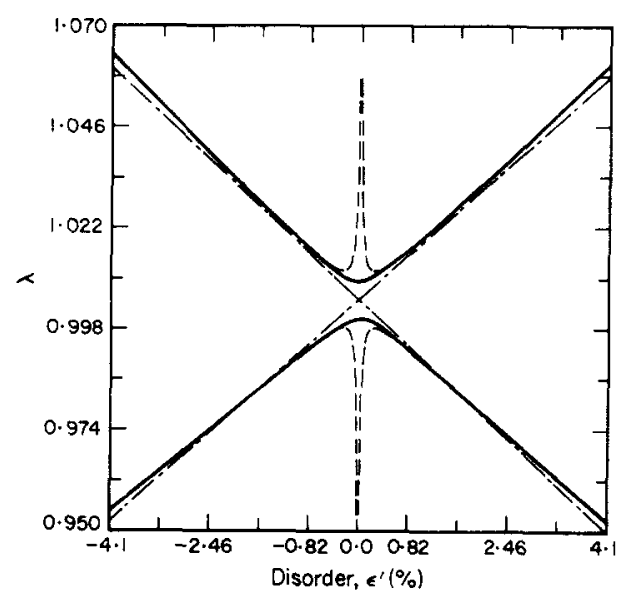

Figure 9. Comparison between the exact method (_-) and the first- (- $\left.-{ }_{-}\right)$and second-order (- -$)^{-}$ modified perturbation methods for the, case of Figure 8.

\section{EXAMPLE 2-TWO-SPAN BEAM}

The loci of the eigenvalues of the two-span beam shown in Figure 4 are considered. The exact solution procedure and the perturbation methods used are outlined in Appendix $B$ and can be found in detail in reference [13]. Due to lack of space, only the lower pair of modes is considered here. Figure 10 displays the loci of the lower two natural frequencies for strong interspan coupling, $c=0$ (no restoring moment at the constraint location), by the exact method. Typical modes of tuned and mistuned systems are also 
shown in Figure 10. Observe that the natural frequencies of the tuned beam are not close, therefore, neither loci veering nor mode localization occurs. On the other hand, Figure 11 is for weak interspan coupling, in which case the tuned eigenvalues are close (the distance between tuned eigenvalues is approximately proportional to 1/c [13]) and, accordingly with the theory of section 3.2 , the natural frequency loci veer away abruptly and the modes, from extended, become strongly localized in one of the spans.

Both classical and modified perturbation methods are outlined in Appendix B for the two-span beam with strong and weak interspan coupling, respectively. In the classical

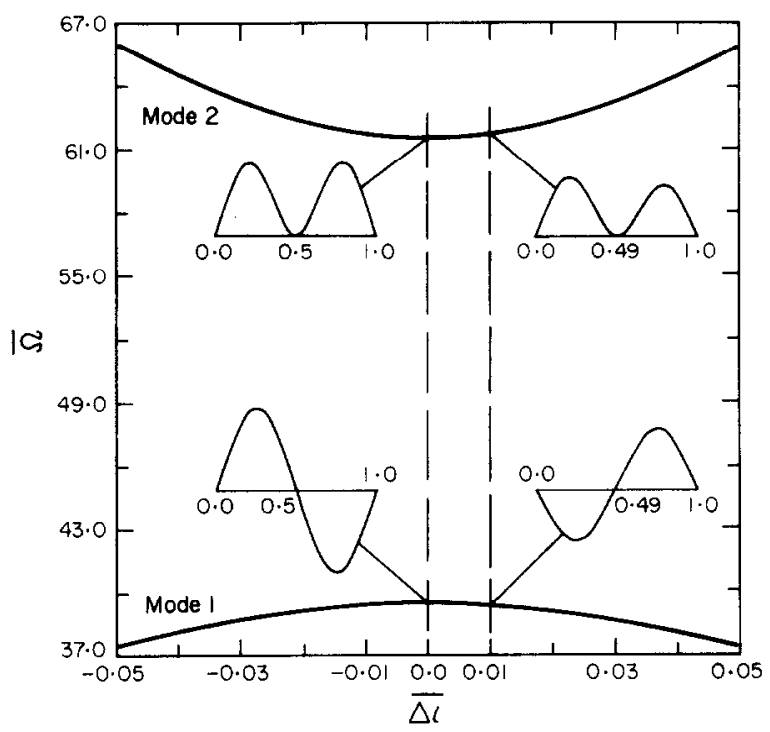

Figure 10. Loci of the lower two dimensionless natural frequencies of a two-span beam with strong interspan coupling, $\bar{c}=0$, versus disorder $\overline{\Delta l}$. Typical mode shapes are represented.

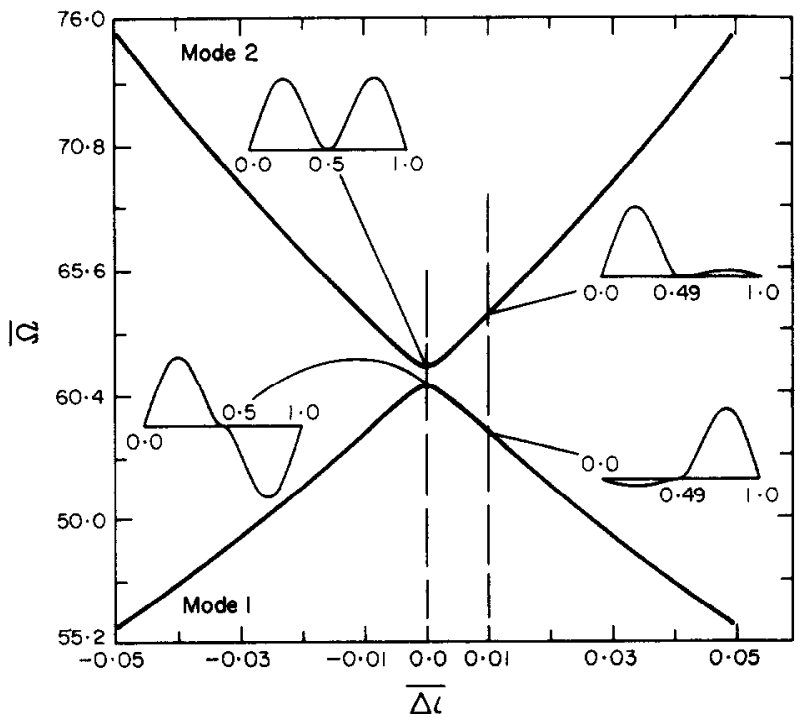

Figure 11. Loci of the lower two frequencies of a two-span beam with weak interspan coupling, $\bar{c}=1000$, versus disorder. Representative modes are shown. 
analysis, the mistuning $\Delta l$ is the perturbation. In the modified case, the unperturbed system consists of two decoupled mistuned spans, and the perturbation is the dimensionless coupling between the spans, $1 / \bar{c}$. Figures 12 and 13 display the comparison between the exact method and the classical and modified perturbation methods in the weak interspan coupling case. Results are similar to the ones of Figures 8 and 9 for a chain of coupled pendulums. Both first- and second-order classical analyses yield erroneous results when localization occurs, but the second order calculation indicates the high sensitivity of the modes to disorder through the detection of the veering as well as describes the loci near the veering. The first-order modified approach gives a good approximation of

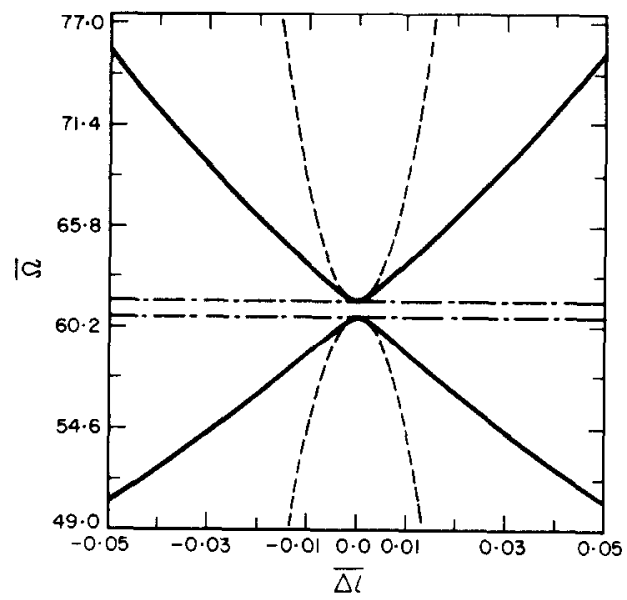

Figure 12. Comparison between the exact method (- - ) and the first- (- - $)$ and second-order (- - ) classical perturbation methods for the case of Figure 11.

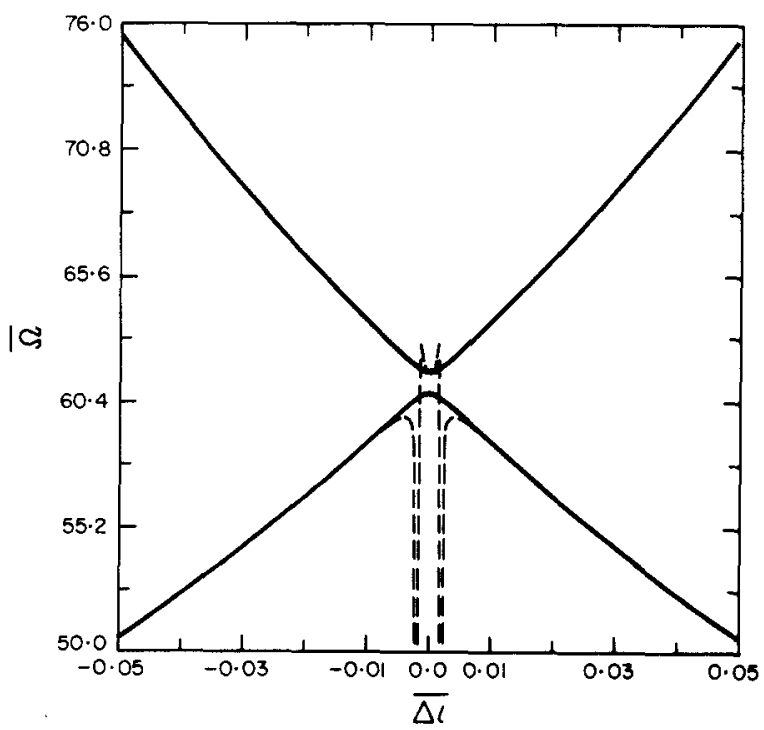

Figure 13. Comparison between the exact method (-) and the first-order modified perturbation method $(---)$ for the case of Figure 11. (Note that the entire dotted line was obtained by perturbation of the first eigenvalue locus, even though this perturbation result approaches the second locus for very small mistuning. For the second eigenvalue locus the agreement between the perturbation and the exact results is so good over the whole mistuning range (even for very small mistuning) that the dotted and solid lines cannot be distinguished.) 
both the eigenvalue loci and the localized modes if mistuning is not too small. If mistuning is very small (of order $\varepsilon^{2}$ ), it is interesting to note that accurate perturbation results are still obtained for the second eigenvalue, while results are erroneous for the first eigenvalue-this is expected, as the classical perturbation method should be used for very small mistuning to describe the veering. Finally, note that the classical first-order eigenvalue perturbation is zero because of symmetry; therefore, the high sensitivity is completely unnoticed by the first-order classical perturbation analysis.

\section{A NOTE ON THE MEMBRANE PROBLEM}

Since loci veering occurs for systems known to be susceptible to mode localization, conversely, one wonders whether some kind of localization always occurs in systems that exhibit curve veering phenomena. The problem of curve veering for a rectangular membrane studied by Leissa [16] and Perkins and Mote [19] is considered. Leissa has shown that when solving for the modes of a clamped rectangular membrane using a three-term Galerkin solution, the loci of $\lambda_{13}$ and $\lambda_{31}$ in terms of the side length ratio, $a / b$, veer away from each other in the neighborhood of the square shape, $a / b=1$. (In this case veering is due to the discretization and does not occur for the exact solution.) This loci veering is displayed in Figure 14, which is taken directly from the literature [16]. Typical corresponding modes are also illustrated by their nodal pattern (lines of zero deflection) in Figure 14. For $a / b=1$, a square membrane, the nodal patterns consist of (approximately) a circle and 2 diagonals, while for $(a / b)^{2}=0.95$ the nodal patterns are two (nearly) horizontal and vertical lines. This drastic change in the modes associated with veering has been analyzed by Leissa $\lceil 16\rceil$.

In light of the discussion of the previous sections, a tuned or ordered membrane can be defined as a square one, and the small difference between the length of the sides can

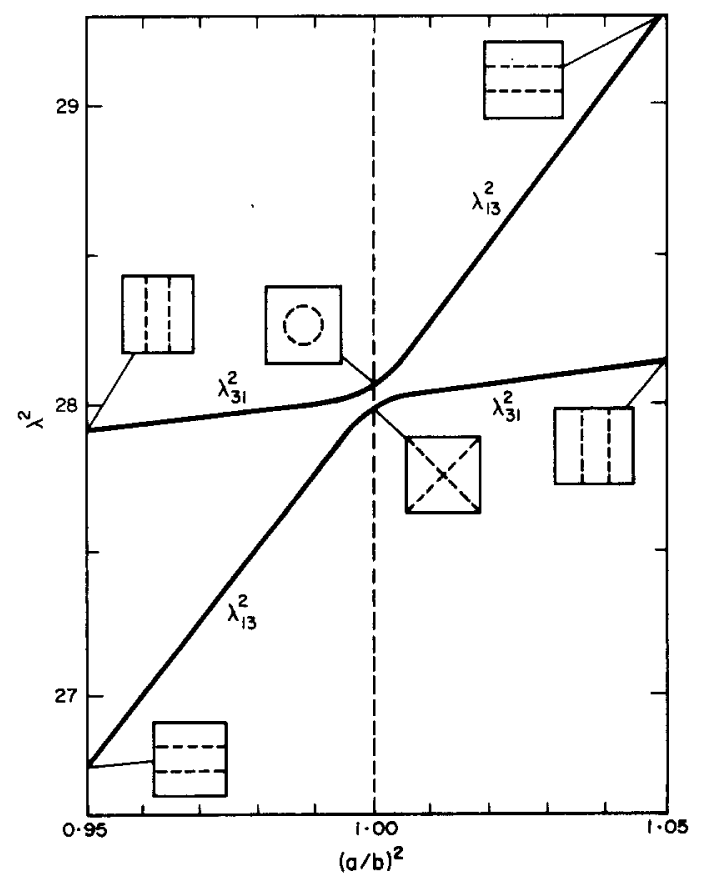

Figure 14. Loci of the square of the natural frequencies of the 13 and 31 modes of a clamped rectangular membrane versus side length ratio, $(a / b)^{2}$. Representative modal patterns are shown $[16,19]$. 
be regarded as disorder. Moreover, it has been shown by Perkins and Mote [19] that, for the discretized solution, disorder couples the mode shapes and therefore results in a veering of the loci. It can be seen in Figure 14 that, in some sense, disorder also results in the "localization" of the modes. The nodal patterns of the tuned membrane (circle and diagonal) extend equally in two directions. On the other hand, each of the nodal lines of the disordered membrane is "localized" in one direction. Therefore, by splitting the eigenvalues of the ordered membrane, the discretization creates a coupling between the modes through the disorder that results in both eigenvalue loci veering and "directional localization." Of course, a classical perturbation method that considers the deviation of the side length ratio from unity as a perturbation could analyze neither the slope change of the loci nor the directional localization of the modes, as it would only be valid in the veering region. Developing a modified perturbation approach would require one to include disorder in the unperturbed system and to consider the distance between unperturbed eigenvalues as a perturbation. This distance, however, results from the Galerkin discretization, and it seems difficult to identify the parameter that governs it. Therefore, it may not be possible to develop such a modified approach for the membrane problem. This note, however, points out that the concepts of disorder and localization indeed apply to other structures than those with spatial periodicity.

\section{CONCLUSIONS}

For self-adjoint free vibration problems, strong mode localization and eigenvalue loci veering are two manifestations of the same drastic phenomenon occurring when some type of disorder is introduced into nearly periodic structures with close eigenvalues.

Classical perturbation methods are useful in predicting this phenomenon. However, it should be borne in mind that first-order eigenvalue perturbation analyses do not reveal the high sensitivity to small disorder, and therefore, second-order eigenvalue perturbation terms that describe the loci in the veering region must be included.

Modified perturbation methods that consider the (small) distance between eigenvalues as a perturbation and include disorder in the unperturbed system effectively analyze the change in slope of the loci due to veering as well as strong localization.

The occurrence of curve veering indicates that, similarly to spatial localization, directional localization also occurs in structures with some kind of irregularities or asymmetry.

\section{ACKNOWLEDGMENT}

This work was supported by NSF Grant No. MSM-8700820, Dynamic Systems and Control Program. Dr Elbert Marsh is the grant monitor.

\section{REFERENCES}

1. P. W. ANDERSON 1958 Physical Review 109, 1492-1505. Absence of diffusion in certain random lattices.

2. E. W. Montroll and R. B. Potts 1955 Physical Review 100, 525-543. Effect of defects on lattice vibrations.

3. H. B. Rosenstock and R. E. MCGILl 1962 Journal of Mathematical Physics 3, 200-202. Vibrational modes of disordered linear chains.

4. H. B. Rosenstock and R. E. MCGILL 1968 Physical Review 176, 1004-1014. Vibrations of disordered solids.

5. K. ISHII 1973 Supplement of the Progress of Theoretical Physics 53, 77-138. Localization of eigenvalues and transport phenomena in the one-dimensional disordered system. 
6. C. H. HODGES 1982 Journal of Sound and Vibration 82(3), 441-424. Confinement of vibration by structural irregularity.

7. C. H. HODGES and J. WoOdHouse 1983 Journal of the Acoustical Society of America 74(3), 894-905. Vibration isolation from irregularity in a nearly periodic structure: theory and measurements.

8. O. O. BENDIKSEN 1984 Proceedings of the XVIth International Congress of Theoretical and Applied Mechanics, Lyngby, Denmark, August 19-25, 1984. Aeroelastic stabilization by disorder and imperfections.

9. N. A. VALERO and O. O. BENDIKSEN 1986 American Society of Mechanical Engineers Journal of Engineering for Gas Turbines and Power 108(2), 293-299. Vibration characteristics of mistuned shrouded blade assemblies.

10. O. O. BENDIKSEN 1987 American Institute of Aeronautics and Astronautics Journal 25(9), 1241-1248. Mode localization phenomena in large space structures.

11. C. PIERRE 1985 Ph.D. Dissertation, Duke University, August 1985. Analysis of structural systems with parameter uncertainties.

12. C. PIERRE and E. H. Dowell 1987 Journal of Sound and Vibration 114, 549-564. Localization of vibrations by structural irregularity.

13. C. Pierre, D. M. TANG and E. H. Dowell 1987 American Institute of Aeronautics and Astronautics Journal 25(9), 1249-1257. Localized vibrations of disordered multi-span beams: theory and experiment.

14. C. PIERRE 1987 Proceedings of the 28th AIAA/ASME/ASCE/AHS Structures, Structural Dynamics and Materials Conference, April 6-8, 1987, Monterey, California. Localized free and forced vibrations of disordered nearly periodic structures.

15. R. A. IbRAHIM 1987 Applied Mechanics Reviews 40(3) 309-328. Structural dynamics with parameter uncertainties.

16. A. W. LeISSA 1974 Journal of Applied Mathematics and Physics (ZAMP) 25, 99-111. On a curve veering aberration.

17. J. R. KUTtLER and V. G. Sigillito 1981 Journal of Sound and Vibration, 75, 585-588. On curve veering.

18. P. S. NAIR and S. DURVASUla 1973 International Journal of Mechanical Sciences 15, 975-986. On quasi-degeneracies in plate vibration problems.

19. N. C. PERKINS and C. D. MOTE JR 1986 Journal of Sound and Vibration 106, 451-463. Comments on curve veering in eigenvalue problems.

20. L. MEIROVITCH 1980 Computational Methods in Structural Dynamics The Netherlands: Sijthoff \& Noordhoff.

21. R. Courant and D. Hilbert 1953 Methods of Mathematical Physics, Volume I. New York: Interscience Publishers, Inc.

22. F. Y. CHEN 1971 Journal of Sound and Vibration 14, 57-79. On modeling and direct solution of certain free vibration systems.

\section{APPENDIX A: CHAIN OF $\boldsymbol{n}$ COUPLED PENDULUMS}

The free vibration eigenvalue problem can be shown to be $[11,12]$

$$
\begin{gathered}
\left([A]-\lambda_{i}[I]\right) \mathbf{x}_{i}=\mathbf{0}, \\
{[A]=\left[\operatorname{tridiag}\left(-R^{2} \frac{1+\Delta l_{i-1}}{1+\Delta l_{i}} ; \frac{1}{1+\Delta l_{i}}+\left(2-\delta_{i}^{n}\right) R^{2} ;-R^{2} \frac{1+\Delta l_{i+1}}{1+\Delta l_{i}}\right)\right] .}
\end{gathered}
$$

$[I]$ is the $n \times n$ identity matrix; $[A]$ is a tridiagonal matrix; $\Delta l_{i}$ is the $i$ th pendulum dimensionless length deviation form the nominal length, $l ; \lambda_{i}=\omega_{i}^{2} /(g / l)$ is a dimensionless eigenvalue, where $\omega_{i}$ is a free vibration natural frequency; $\mathbf{x}_{i}$ is the corresponding eigenvector of $n$ angular amplitudes $\theta_{k}, k=1, \ldots, n$; and $R^{2}=(k / m) /(g / l)$ is the dimensionless coupling. The other parameters are defined in Figure 3 . The tuned eigensolution is [22]

$$
\begin{gathered}
\lambda_{0 i}=1+2 R^{2}\left[1-\cos \frac{(2 i-1) \pi}{2 n+1}\right], \quad \mathbf{x}_{0 i}=\left[\sin \frac{(2 i-1) \pi}{2 n+1}, \ldots, \sin \frac{n(2 i-1) \pi}{2 n+1}\right]^{\mathrm{T}}, \\
i=1, \ldots, n .
\end{gathered}
$$


Classical perturbation method for strong coupling. The $\Delta l_{i}$ 's are the perturbations. The unperturbed eigensolution is given by equations (A2). The unperturbed and first-order perturbation matrices are

$$
\begin{aligned}
{\left[A_{0}^{c}\right] } & =\left[\operatorname{tridiag}\left(-R^{2} ; 1+\left(2-\delta_{i}^{n}\right) R^{2} ;-R^{2}\right)\right], \\
{\left[\delta A^{c}\right] } & =\left[\operatorname{tridiag}\left(-R^{2}\left(\Delta l_{i-1}-\Delta l_{i}\right) ;-\Delta l_{i} ;-R^{2}\left(\Delta l_{i+1}-\Delta l_{i}\right)\right)\right] .
\end{aligned}
$$

Modified perturbation method for weak coupling. The $\Delta l_{i}$ 's are included in the unperturbed system; $R^{2}$ is the perturbation. The unperturbed and perturbation matrices are

$$
\begin{gathered}
{\left[A_{0}^{m}\right]=\left[\operatorname{diag}\left(1-\Delta l_{i}\right)\right], \quad\left[\delta A^{m}\right]=\left[\operatorname{tridiag}\left(-R^{2} ;\left(2-\delta_{i}^{n}\right) R^{2} ;-R^{2}\right)\right],} \\
{\left[\delta^{2} A^{m}\right]=\left[\operatorname{tridiag}\left(R^{2}\left(\Delta l_{i}-\Delta l_{i-1}\right) ; \Delta l_{i}^{2} ; R^{2}\left(\Delta l_{i}-\Delta l_{i+1}\right)\right)\right] .}
\end{gathered}
$$

The unperturbed eigensolution is

$$
\lambda_{0 i}^{m}=1-\Delta l_{i}, \quad \mathbf{x}_{0 i}^{m}=\mathbf{e}_{i} \text { (canonical vector) }
$$

\section{APPENDIX B: TWO-SPAN BEAM}

The system shown in Figure 4 is analyzed by a Rayleigh-Ritz procedure detailed in reference [13]. The transverse deflection is expanded in terms of the modes of a single-span beam of length $l$ pinned at both ends, with the constraint conditions enforced by means of Lagrange multipliers. The frequency equation can be shown to be

$\left\{\sum_{i=1}^{N M} \frac{\sin ^{2}\left(i \pi \bar{x}_{1}\right)}{(i \pi)^{4}-\bar{\Omega}^{2}}\right\}\left\{\frac{1}{\bar{c}}+\sum_{i=1}^{N M} \frac{(i \pi)^{2} \cos ^{2}\left(i \pi \bar{x}_{1}\right)}{(i \pi)^{4}-\bar{\Omega}^{2}}\right\}-\left\{\sum_{i=1}^{N M} \frac{(i \pi) \sin \left(i \pi \bar{x}_{1}\right) \cos \left(i \pi \bar{x}_{1}\right)}{(i \pi)^{4}-\bar{\Omega}^{2}}\right\}^{2}=0$.

$\bar{\Omega}=\Omega / \sqrt{E I / m l^{4}}$ is the dimensionless frequency, where $E I$ is the bending stiffness and $m$ the mass per unit length; $N M$ is the number of component modes in the Rayleigh-Ritz procedure; and $\bar{x}_{1}=\frac{1}{2}-\overline{\Delta l}$ is the dimensionless location of the intermediate support, where $\overline{\Delta l}=\Delta l / l ; \bar{c}=2 \mathrm{cl} / E I$ is the dimensionless torsional spring constant.

Classical perturbation method for strong interspan coupling. The unperturbed system is the tuned beam and the perturbation is $\overline{\Delta l}$. One has

$$
\bar{\Omega}=\bar{\Omega}_{0}+\delta \bar{\Omega}+\delta^{2} \bar{\Omega}+\text { H.O.T. }
$$

where $\bar{\Omega}_{0}$ is the solution of equation (B1) for $\overline{\Delta l}=0$, and $\delta \bar{\Omega}$ and $\delta^{2} \bar{\Omega}$ are first- and second-order perturbations in $\overline{\Delta l}$. The substitution of expression (B2) into equation (B1) and subsequent expansion yields

$$
\delta \bar{\Omega}=0, \quad \delta^{2} \bar{\Omega}=-(\alpha / \beta)\left(\overline{\Delta l}^{2} / 2 \bar{\Omega}_{0}\right),
$$

where

$$
\begin{gathered}
\alpha=\sum_{i \text { odd }} \frac{1}{z_{i}} \sum_{i=1}^{N M} \frac{(-1)^{i+1}(i \pi)^{4}}{z_{i}}-\left\{\sum_{i=1}^{N M} \frac{(-1)^{i}(i \pi)^{2}}{z_{i}}\right\}^{2}+\sum_{i=1}^{N M} \frac{(-1)^{i}(i \pi)^{2}}{z_{i}}\left\{\frac{1}{\bar{c}}+\sum_{i \text { even }} \frac{(i \pi)^{2}}{z_{i}}\right\}, \\
\beta=\sum_{\text {i odd }} \frac{1}{z_{i}} \sum_{\text {ieven }} \frac{(i \pi)^{2}}{z_{i}^{2}}+\sum_{i \text { odd }} \frac{1}{z_{i}^{2}}\left[\frac{1}{\bar{c}}+\sum_{i \text { even }} \frac{(i \pi)^{2}}{z_{i}}\right],
\end{gathered}
$$

and $z_{i}=(i \pi)^{4}-\bar{\Omega}_{0}^{2}$

Modified perturbation method for weak interspan coupling. $\overline{\Delta l}$ is included in the unperturbed system and $1 / \bar{c}$ is a perturbation. $\bar{\Omega}_{0}^{m}$ are the natural frequencies of pinned-clamped spans of rengths $\frac{1}{2}-\overline{\Delta l}$ and $\frac{1}{2}+\overline{\Delta l}$, obtained by solving equation (B1) for $1 / \bar{c}=0 . \delta \bar{\Omega}^{m}$ is the first-order perturbation in $1 / \bar{c}$. One obtains [13]

$$
\delta \bar{\Omega}^{m}=\frac{1}{\bar{c}} \frac{-1}{2 \bar{\Omega}_{0}^{m} \gamma} \sum_{i=1}^{N M} \frac{\sin ^{2}\left(i \pi \bar{x}_{1}\right)}{y_{i}},
$$


where

$$
\begin{aligned}
\gamma= & \sum_{i=1}^{N M} \frac{\sin ^{2}\left(i \pi \bar{x}_{1}\right)}{y_{i}} \sum_{i=1}^{N M} \frac{(i \pi)^{2} \cos ^{2}\left(i \pi \bar{x}_{1}\right)}{y_{i}^{2}}+\sum_{i=1}^{N M} \frac{\sin ^{2}\left(i \pi \bar{x}_{1}\right)}{y_{i}^{2}} \sum_{i=1}^{N M} \frac{(i \pi)^{2} \cos ^{2}\left(i \pi \bar{x}_{1}\right)}{y_{i}} \\
& -2 \sum_{i=1}^{N M} \frac{(i \pi) \sin \left(i \pi \bar{x}_{1}\right) \cos \left(i \pi \bar{x}_{1}\right)}{y_{i}} \sum_{i=1}^{N M} \frac{(i \pi) \sin \left(i \pi \bar{x}_{1}\right) \cos \left(i \pi \bar{x}_{1}\right)}{y_{i}^{2}}
\end{aligned}
$$

and $y_{i}=(i \pi)^{4}-\left(\bar{\Omega}_{0}^{m}\right)^{2}$. 